PROCEEDINGS OF THE

AMERICAN MATHEMATICAL SOCIETY

Volume 137, Number 11, November 2009, Pages 3757-3762

S 0002-9939(09)09938-9

Article electronically published on May 28, 2009

\title{
SUBSTRICTLY CYCLIC OPERATORS
}

\author{
BEN MATHES \\ (Communicated by Nigel J. Kalton) \\ Dedicated to Don Hadwin
}

\begin{abstract}
We initiate the study of substrictly cyclic operators and algebras. As an application of this theory, we are able to give a description of the strongly closed ideals in the commutant of the Volterra operator, and quite a bit more.
\end{abstract}

\section{INTRODUCTION}

Strictly cyclic algebras were introduced by Lambert (see 9 and [10]) and studied extensively by Herrero in the papers [5] and [6. A brief survey may be found in 14 . Let $\mathcal{H}$ denote a complex Hilbert space and assume that there is a unital abelian multiplication defined on $\mathcal{H}$ that satisfies

$$
\|x y\| \leq k\|x|||| y\|
$$

for some constant $k$ and all $x, y \in \mathcal{H}$. Each element $x$ of $\mathcal{H}$ may then be thought of as an operator $M_{x}$ on $\mathcal{H}$, via

$$
M_{x} y=x y,
$$

and the map $M: \mathcal{H} \rightarrow B(\mathcal{H})$ is a bounded invertible isomorphism of $\mathcal{H}$ with the algebra of multipliers, whose inverse is evaluation at the unit. The algebra of multipliers is what people have called a strictly cyclic algebra, and if the algebra is singly generated, the multiplier corresponding to any generator has been called a strictly cyclic operator.

If an abelian algebra has a strict cyclic vector, i.e. a vector $e$ such that evaluation at $e$ maps the algebra onto the Hilbert space, then the evaluation at $e$ will also be injective, so the multiplication on the algebra may be transported to the Hilbert space, and we see that every abelian strictly cyclic algebra is an algebra of multipliers as described above.

\section{Substrictly CyCliC Algebras}

We assume now that $\mathcal{H}$ has a bounded abelian multiplication that may or may not be unital, a structure we call a Hilbert ring. We again let $M_{x}$ denote the multiplier that corresponds to a vector $x \in \mathcal{H}$. The mapping $M: \mathcal{H} \rightarrow B(\mathcal{H})$ is still bounded, but it may not be bounded below. We will refer to the strong closure

Received by the editors April 29, 2008, and, in revised form, February 20, 2009.

2000 Mathematics Subject Classification. Primary 46B28, 47L50, 46L07; Secondary $46 \mathrm{H} 10$.

Key words and phrases. Strictly cyclic algebra, strictly cyclic operators, large point spectrum.

(C)2009 American Mathematical Society Reverts to public domain 28 years from publication 
of the range of $M$ as a substrictly cyclic algebra. If $x$ is a generator in $\mathcal{H}$, then we will call $M_{x}$ a substrictly cyclic operator.

We will consistently use techniques of Banach algebra theory on our Hilbert ring $\mathcal{H}$, even though $\mathcal{H}$ is not technically a Banach algebra. This is not a problem, however, since there is an equivalent norm on $\mathcal{H}$ that does make it into a Banach algebra. One way to get such a norm is to unitize $\mathcal{H}$, after which the unital Hilbert ring will be isomorphic (as a Banach space) to its algebra of multipliers, which is a Banach algebra.

Proposition 2.1. Assume that the operator $T$ is substrictly cyclic. Then there exists a strictly cyclic operator $S$ such that $T$ is the restriction of $S$ to a maximal invariant subspace.

Proof. Assume that $T=M_{x}$, where $x$ is a generator in $\mathcal{H}$. We adjoin a unit element to $\mathcal{H}$ in the traditional manner, defining a multiplication on $\mathbb{C} \oplus \mathcal{H}$ by

$$
(\alpha, a)(\beta, b)=(\alpha \beta, \alpha b+\beta a+a b)
$$

and we refer to this as the unitization of $\mathcal{H}$. It follows that $T$ is the restriction of the strictly cyclic operator $M_{(0, x)}$ to the maximal invariant subspace $0 \oplus \mathcal{H}$.

Example 2.1. Let $\mathcal{H}$ be a Hilbert space with the trivial multiplication:

$$
x y=0
$$

for every $x, y \in \mathcal{H}$. This example shows that the multiplier map can be zero, as far from injective as we can get. The unitization gives a strictly cyclic algebra whose operators have the form

$$
\left[\begin{array}{cc}
a & 0 \\
x & a I
\end{array}\right],
$$

where $a \in \mathbb{C}$ and $x \in \mathcal{H}$. This is Lambert's example in [9] of an abelian strictly cyclic algebra that is not singly generated.

We will need the following analogue of the well-known fact that the compression of a strictly cyclic operator to a closed complement of an invariant subspace is strictly cyclic [6].

Proposition 2.2. Assume that $T$ is substrictly cyclic and that $\mathcal{I}$ is a closed ideal in $\mathcal{H}$. Then the compression of $T$ to a closed complement of $\mathcal{I}$ is substrictly cyclic.

Proof. Assume $\mathcal{M}$ is a closed complement of $\mathcal{I}$ and let $P$ denote the bounded idempotent that projects onto $\mathcal{M}$ in the direction of $\mathcal{I}$. Let $x$ be a generator of $\mathcal{H}$ such that $M_{x}=T$. It follows that $M_{x+\mathcal{I}}$ is a substrictly cyclic operator acting on the quotient Hilbert ring $\mathcal{H} / \mathcal{I}$. The mapping $\Gamma$ that takes $a \in \mathcal{M}$ to $a+\mathcal{I}$ is contractive, and it has a bounded inverse, so it is a Banach space isomorphism. Thus we may endow $\mathcal{M}$ with a bounded multiplication by using $\Gamma^{-1}$ to carry the multiplication of $\mathcal{H} / \mathcal{I}$ over to $\mathcal{M}$. This done, we see that a generator is $\Gamma^{-1}(x+\mathcal{I})=P x$, and the fact that $\mathcal{I}$ is an ideal gives us

$$
P M_{x}(a)=P M_{P x}(a)
$$

for every $a \in \mathcal{M}$. Thus the compression of $T$ to $\mathcal{M}$ is the multiplier corresponding to the generator $P x$. 
Example 2.2. Let $e_{i}$ be an orthonormal basis of $\mathcal{H}$ and define the multiplication coordinatewise:

$$
x y=\sum\left\langle x, e_{i}\right\rangle\left\langle y, e_{i}\right\rangle e_{i} .
$$

Then $\mathcal{H}$ is unital exactly when it is finite dimensional. In the infinite dimensional case, the unitization of this Hilbert ring is the strictly cyclic algebra studied in [7] and in 3. In this example, the generators of the Hilbert ring are vectors with distinct (nonrepeating) coordinates. The multipliers $M_{x}(x \in \mathcal{H})$ are the diagonal Hilbert-Schmidt operators, and with our terminology, the algebra of all bounded diagonal operators is the substrictly cyclic algebra. The substrictly cyclic operators are the generators of the algebra of diagonal operators that are Hilbert-Schmidt. This example is actually a model for all of the normal substrictly cyclic operators, and it might be contrasted with the results in 1 .

Proposition 2.3. A normal operator is substrictly cyclic if and only if it is HilbertSchmidt with distinct eigenvalues.

Proof. We assert that every normal substrictly cyclic operator has an eigenvector. To see this, assume $\mathcal{H}$ is a Hilbert ring, $x \in \mathcal{H}$ is a generator, and $M_{x}$ is normal. The spectral radius of $M_{x}$ equals its norm and is thus nonzero. Hence there is a multiplicative functional $y \in \mathcal{H}$ such that

$$
\left\langle u, M_{x}^{*} y\right\rangle=\langle x u, y\rangle=\langle x, y\rangle\langle u, y\rangle=\langle u,\langle y, x\rangle y\rangle,
$$

so $y$ is an eigenvector for $M_{x}^{*}$, and hence also for $M_{x}$.

Assume now that $\mathcal{E}$ is a maximal orthonormal set of eigenvectors for $M_{x}$; we will show it must be a basis. The subspace $\mathcal{E}^{\perp}$ is reducing for $M_{x}$, and by Proposition 2.2. the restriction of $M_{x}$ to $\mathcal{E}^{\perp}$ will also be a normal substrictly cyclic operator. If this restriction is not zero, then the restriction must have an eigenvector, contradicting the maximality of $\mathcal{E}$.

We will say that $\mathcal{H}$ is a Hilbert ring with approximate identity if there exists a net $i_{\lambda}$ in $\mathcal{H}$ such that $i_{\lambda} x \rightarrow x$ for every $x \in \mathcal{H}$. If the multipliers $M_{i_{\lambda}}$ are bounded, we will say that we have a bounded approximate identity, and if the multipliers are all contractions, we will say that we have a contractive approximate identity. It is often the case that an approximate identity is not bounded relative to the Hilbert norm on $\mathcal{H}$, but it can still be a bounded or contractive approximate identity with our definition.

Example 2.3. Let $\mathcal{H}$ be $L^{2}(0,1)$, and define the convolution multiplication

$$
f \circ g(x)=\int_{0}^{x} f(s) g(x-s),
$$

which turns $\mathcal{H}$ into a Hilbert ring with approximate identity

$$
i_{t}(x)=(1 / t) \chi_{[0, t]},
$$

where $\chi_{S}$ denotes the characteristic function of the set $S$ (see [8, p. 400). This is a singly generated Hilbert ring, one of whose generators is $\chi_{[0,1]}$ with the corresponding substrictly cyclic multiplier

$$
M_{\chi_{[0,1]}} f(x)=V f(x)=\int_{0}^{x} f(s) d s,
$$

the classical Volterra operator. 
Proposition 2.4. If $\mathcal{H}$ is a Hilbert ring with approximate identity, then the corresponding substrictly cyclic algebra is maximal abelian. Moreover, if $\mathcal{H}$ is singly generated with generator $x$, then the strongly closed algebra generated by $M_{x}$ and the identity is the same as the strongly closed algebra generated by $M_{x}$.

Proof. Assume $A$ commutes with every multiplier. Then $A M_{i_{t}}=M_{A\left(i_{t}\right)}$ converges to $A$ in the strong operator topology, so $A$ is an element of the substrictly cyclic algebra. For the second assertion, note that the strongly closed algebra generated by $M_{x}$ contains all of the multipliers, and in particular it contains $M_{i_{t}}$, which converge strongly to the identity operator.

We will say that a norm closed algebra $\mathcal{A}$ has the Kaplansky density property if, given every contraction $T$ in the strong closure $\mathcal{A}^{-s o t}$ of $\mathcal{A}, T$ is a strong limit of contractions in $\mathcal{A}$. We will say that a set of operators $\mathcal{X}$ has the Kaplansky density property (or more briefly the density property) if the norm closed algebra generated by $\mathcal{X}$ has it. If the set contains a single $T$ and has the density property, then we say $T$ has the density property.

If an algebra has the density property, then the strong closure must equal the weak-* closure. This is because the strong closure of an algebra equals the weak operator closure, and the weak-* topology coincides with the weak operator topology on bounded sets. Wogen gave an example in [16] of an operator $T$ for which the strongly closed algebra generated by $T$ is different than the weak-* closed algebra generated by $T$, so this operator also gives an example of one that fails the density property.

Proposition 2.5. If $\mathcal{H}$ is a Hilbert ring with a contractive approximate identity, then the norm closure of the set of multipliers has the Kaplansky density property. In particular, if $\mathcal{H}$ is singly generated, then the multipliers corresponding to the generators are substrictly cyclic operators with the Kaplansky density property.

Proof. If $A$ is a contraction in the substrictly cyclic algebra, then

$$
A M_{i_{t}}=M_{A\left(i_{t}\right)}
$$

are contractions converging strongly to $A$.

Sarason proved in [13] that the algebra generated by the Volterra operator is maximal abelian. A simple proof of this result was later given by Erdos in 4, and slightly strengthened to show that the strongly closed algebra generated by $V$ is the same as the strongly closed algebra generated by $V$ and the identity operator. Erdos' proof is given by Davidson in 2], where it is emphasized that the Volterra operator has the density property. We recapture all of these results as a corollary.

Corollary 2.4. The Volterra operator $V$ has the density property, and the strongly closed algebra generated by $V$ is maximal abelian, hence equal to the commutant of $V$.

Proof. The approximate identity given in Example 2.3 is a contractive approximate identity, since $\left\|i_{t} \circ x\right\|_{2} \leq\left\|i_{t}\right\|_{1}\|x\|_{2}=\|x\|_{2}$ (see [11], page 322).

Example 2.5. In [12] Sarason characterizes the invariant subspaces of

$$
M_{x}+V
$$


where $V$ is the Volterra operator and $M_{x}$ is multiplication by $x$ on $L^{2}(0,1)$. He does this by using the Volterra operator to transport the problem to the algebra of absolutely continuous functions on the unit interval and by endowing the algebra with a norm in such a way that the Volterra operator becomes an isometry. After doing so, multiplication by $x$ on the algebra of absolutely continuous functions that vanish at zero is unitarily equivalent to $M_{x}+V$, so this is a substrictly cyclic operator. Instead of carrying the norm over to the absolutely continuous functions, we could carry the multiplication back to $L^{2}(0,1)$, where it takes the form

$$
f \star g=(V f) g+(V g) f
$$

and when we do we see that $i_{t}(x)=(1 / t) \chi_{[0, t]}$ is also an approximate identity for this ring. This time it fails to be a contractive approximate identity, but it is a bounded approximate identity. We thus are able to get corollaries for this operator. In [15] Waterman worked with an algebra unitarily equivalent to this substrictly cyclic algebra and showed that it was maximal abelian, so our corollary recaptures this result and adds a little bit more.

Corollary 2.6. The strongly closed algebra generated by $M_{x}+V$ equals the strongly closed algebra generated by $M_{x}+V$ and the identity, and this substrictly cyclic algebra is maximal abelian. Moreover, since the approximate identity is bounded, the weak-* closed algebra generated by $M_{x}+V$ equals the strongly closed algebra generated by $M_{x}+V$.

\section{Strongly Closed IDEALS}

We now set out to characterize the strongly closed ideals in the commutant of the Volterra operator. Let $\mathcal{H}$ denote the Volterra Hilbert ring; i.e., it denotes $L^{2}(0,1)$ with the convolution multiplication. The closed ideals in $\mathcal{H}$ are precisely the invariant subspaces of the Volterra operator, so they constitute a chain $\mathcal{I}_{t}$ with $0<t<1$, where $\mathcal{I}_{t}$ is the subspace of functions that vanish almost everywhere on $(0, t)($ see 12$])$.

Let $\mathcal{A}$ denote the strongly closed algebra generated by the Volterra operator, and let $\mathcal{J}_{t}$ denote the strong closure of the set of multipliers $M_{x}$ with $x \in \mathcal{I}_{t}$. If $\mathcal{J}$ is any strongly closed ideal in $\mathcal{A}$, then $M^{-1} \mathcal{J}$ is a closed ideal in $\mathcal{H}$. If $A \in \mathcal{J}$ is a nonzero element and $x \in \mathcal{H}$ is taken so that $A x \neq 0$, then $A M_{x}=M_{A x} \in \mathcal{J}$, and it follows that $M^{-1} \mathcal{J}$ is a proper ideal, i.e. that

$$
M^{-1} \mathcal{J}=\mathcal{I}_{t}
$$

for some $t \in(0,1)$. It is clear that $\mathcal{J}_{t} \subseteq \mathcal{J}$, and since $M_{A i_{t}}$ converges strongly to $A$ we see that

$$
\mathcal{J}_{t}=\mathcal{J}
$$

so every strongly closed ideal is of the form $\mathcal{J}_{t}$.

If $S \subseteq \mathcal{H}$, then we denote its annihilator ideal by ann $S$, and it is defined by

$$
\operatorname{ann} S=\{x \in \mathcal{H}: x y=0 \text { for all } y \in S\} .
$$

The annihilator ideal of a subset of $\mathcal{A}$ will be denoted in the same way. Notice that, for every $t \in(0,1)$,

$$
\operatorname{ann} \mathcal{I}_{t}=\mathcal{I}_{1-t} \text { and } \operatorname{ann} \mathcal{J}_{t}=\mathcal{J}_{1-t}
$$


and the map that takes $\mathcal{I}_{t}$ to $\mathcal{J}_{t}$ is injective. More generally, one sees that $A \in \mathcal{J}_{s}$ and $B \in \mathcal{J}_{t}$ imply $A B \in \mathcal{J}_{s+t}$ if we adopt the convention that $\mathcal{J}_{t}$ is the zero ideal when $t \geq 1$. We record these observations in our final

Proposition 3.1. The strongly closed ideals of the commutant of the Volterra operator form a chain $\mathcal{J}_{t}$ with $t \in(0,1)$ such that the annihilator of $\mathcal{J}_{t}$ is $\mathcal{J}_{1-t}$. In particular, each ideal consists entirely of nilpotent elements, $\mathcal{J}_{1 / 2}$ consists entirely of square zero nilpotent elements, and a nilpotent element of any order $n$ may be found in an appropriate ideal.

\section{REFERENCES}

1. R. Bolstein, W. Wogen, Subnormal operators in strictly cyclic operator algebras, Pacific J. Math. 49 (1973), 7-11. MR.0355670 (50:8144)

2. K. Davidson, Nest Algebras, Pitman Research Notes in Mathematics Series 191 (Longman Scientific and Technical, 1988). MR972978 (90f:47062)

3. J. Froelich, B. Mathes, Bi-strictly cyclic operators, New York J. Math. 1 (1995), 97-110. MR.1323805 (96h:47009)

4. J. A. Erdos, The commutant of the Volterra operator, Integral Equations Operator Theory $\mathbf{5}$ (1982), 127-130 MR646883 (83i:47040)

5. D. A. Herrero, Operator algebras of finite strict multiplicity, Indiana Univ. Math. J. 22 (1972), 13-24. MR0315468 (47:4017)

6. D. A. Herrero, Operator algebras of finite strict multiplicity. II, Indiana Univ. Math. J. 27 (1978), 9-18. MR475718 (81g:47048)

7. D. A. Herrero, D. R. Larson, W. R. Wogen, Semitriangular operators, Houston J. Math. 17 (1991), 477-499. MR.1147270 (92m:47037)

8. E. Hewitt, K. Stromberg, Real and Abstract Analysis, Graduate Texts in Mathematics 25, Springer-Verlag, 1965. MR0367121 (51:3363)

9. A. Lambert, Strictly Cyclic Operator Algebras, Dissertation, University of Michigan, Ann Arbor, Mich., 1970.

10. A. Lambert, Strictly cyclic operator algebras, Pacific J. Math. 39 (1971), 717-726. MR0310664 $(46: 9762)$

11. C. Rickart, General Theory of Banach Algebras, The University Series in Higher Mathematics, Van Nostrand, 1960. MR0115101 (22:5903)

12. D. Sarason, Invariant Subspaces, Topics in Operator Theory, Math. Surveys, no. 13, Amer. Math. Soc., Providence, R.I., 1974. MR.0358396 (50:10862)

13. D. Sarason, Generalized interpolation in $H^{\infty}$, Trans. Amer. Math. Soc. 127 (1967), 179-203. MR $0208383(34: 8193)$

14. A. Shields, Weighted shift operators and analytic function theory, Topics in Operator Theory, Math. Surveys, no. 13, Amer. Math. Soc., Providence, R.I., 1974. MR0361899 (50:14341)

15. R. E. Waterman, Invariant subspaces, similarity and isometric equivalence of certain commuting operators in $L_{p}$, Pacific J. Math. 48 (1973), 593-613. MR0399894 (53:3735)

16. W. R. Wogen, Some counterexamples in nonselfadjoint algebras, Ann. of Math. (2) 126 (1987), 415-427. MR.908152 (89b:47066)

Department of Mathematics, Colby College, Waterville, Maine 04963

E-mail address: dbmathes@colby.edu 